\title{
Preliminary Recommendations for Surgical Practice of Neurosurgery Department in the Central Epidemic Area of 2019 Coronavirus Infection
}

\author{
Yu-tang TAN, Jun-wen WANG, Kai ZHAO, Lin HAN, Hua-qiu ZHANG, Hong-quan NIU, Kai SHU\#, Ting LEI \\ Department of Neurosurgery, Tongji Hospital, Tongji Medical College, Huazhong University of Science and Technology, \\ Wuhan 430030, China
}

(C) Huazhong University of Science and Technology 2020

\begin{abstract}
Summary: Since December 2019, an outbreak of coronavirus disease 2019 (COVID-19) has posed significant threats to the public health and life in China. Unlike the other 6 identified coronaviruses, the SARS-Cov-2 has a high infectious rate, a long incubation period and a variety of manifestations. In the absence of effective treatments for the virus, it becomes extremely urgent to develop scientific and standardized proposals for prevention and control of virus transmission. Hereby we focused on the surgical practice in Neurosurgery Department, Tongji Hospital, Wuhan, and drafted several recommendations based on the latest relevant guidelines and our experience. These recommendations have helped us until now to achieve 'zero infection' of doctors and nurses in our department, we would like to share them with other medical staff of neurosurgery to fight 2019-nCoV infection.
\end{abstract}

Key words: SARS-CoV-2; Neurosurgery; Diagnosis; Treatment; Prevention

\section{INTRODUCTION}

Since December 2019, several cases of atypical pneumonia have been reported in Wuhan. On January 2020, a novel coronavirus was identified by the Chinese Center for Disease Control and Prevention (CDC) from the throat swab sample of pneumonia patients, and was named coronavirus disease 2019 (COVID-19) by World Health Organization (WHO) ${ }^{[1,2]}$. By 28 February 2020, nearly 78959 cases of COVID-19 have been confirmed nationwide with another 2308 cases suspected.

In the last decades, 6 coronavirus species (CoVs) have shown to be capable of infecting humans as well as their regular intermediate hosts, such as birds and mammals. The outbreak of severe acute respiratory syndrome coronavirus (SARS) and Middle East respiratory syndrome coronavirus (MERS) in 2012 has revealed the lethality of CoVs when they infect humans. SARS-CoV-2 is the 7th identified member of CoVs family ${ }^{[3-5]}$. Phylogenetic genome sequencing has shown that SARS-CoV-2 was most closely related (89.1\% similarity) to a group of SARS-like $\mathrm{CoVs}^{[6]}$.

Retrospective studies revealed that the typical clinical manifestations were fever, cough, and dyspnea. However, a number of patients only presented initially

\footnotetext{
Yu-tang TAN, E-mail: 5364478@qq.com \#Corresponding authors, Kai SHU, E-mail: Kshu@tjh.tjmu. edu.cn; Ting LEI, E-mail: tlei@tjh.tjmu.edu.cn
}

with gastrointestinal symptoms. Evidence has shown that human-to-human transmission of SARS-CoV-2 had occurred by close contacts or through virus-laden aerosols ${ }^{[7,8]}$. The incubation period ranged from 3 days to 14 days $^{[9,10]}$. Patients may develop a rapid and serious dyspnea in a few days after infection. The mortality was about $3.5 \%$ according to the latest government report.

Although WHO suggested that the current event did not constitute a Public Health Emergency of International Concern (PHEIC), the COVID-19 situation exerted significant threats to public health and life in China ${ }^{[11,12]}$. Fortunately, the Chinese government made a rapid and effective response to this situation and made a great progress in protecting citizens from virus transmission. Wuhan City became the battlefront to fight COVID-19, and most doctors devoted themselves to this white war. Under novel coronavirus pneumonia situation, regular clinical work in the local hospital was seriously restricted. Especially, it became much more challenging to deal with the surgical requests for patients with central nervous system (CNS) diseases. As the largest neurosurgical center in Wuhan, Neurosurgery Department of Tongji Hospital performed surgical treatments for patients in the epidemic situation. We carried out some management proposals of the patients on the basis of conventional treatment guidelines and clinical experiences. These recommendations have helped us until now to achieve 'zero infection' of doctors and nurses in our department, we would like to 
share with other medical staff of neurosurgery to fight novel coronavirus infection.

\section{RECOMMENDATIONS FOR THE MANAGE- MENT OF PATIENTS WITH CNS DISEASES}

\subsection{Preoperative Evaluation And Management}

All patients were first applied to the special fever clinics in out-patient department. After temperature test, a careful history query (especially the fever and cough manifestations in the last 2 weeks) and physical examination were performed by doctors from both outpatient and neurosurgery departments under strict third level protection (medical masks, protective goggles and suit). Surgical indications should be rigorously evaluated and surgical treatment should be preserved for patients with emergency condition, such as ruptured aneurysm and intracranial hemorrhage. Operations for patients with relatively stable condition should be postponed, for example, patients with benign brain tumors. These patients were documented and followedup through phone call.

A pulmonary computed tomography (CT) scan and nucleic acid sequencing of throat swab were recommended for preliminary diagnosis of COVID-19 infection before hospitalization. Patients with positive results were identified to be confirmed cases and patients with preliminary negative results were considered to be suspected cases. However, these examinations should be canceled and a direct emergency surgery should be performed for patients under life-threating condition. Patients without immediate life-threating were transferred to the neurosurgery ward through special lane to avoid cross infection.

The neurosurgery ward was divided into several areas: patient rooms were regarded as infected area, while nurse station and doctor office were considered to be clean area. Patient rooms were further divided into two partitions for suspected cases and confirmed cases. Individual accommodation was recommended for all patients and rigorous quarantine should be applied to the confirmed cases. Daily sterilization was performed for each single room. Doctors and nurses must take strict third level protection before entering patient rooms. Regular preoperative neuroimaging and laboratory examinations were performed after hospitalization. We must emphasize that a consultation from anesthesiologists and perioperative nurses was necessary to decide the date of operation and the intraoperative cooperation strategies (fig. 1).

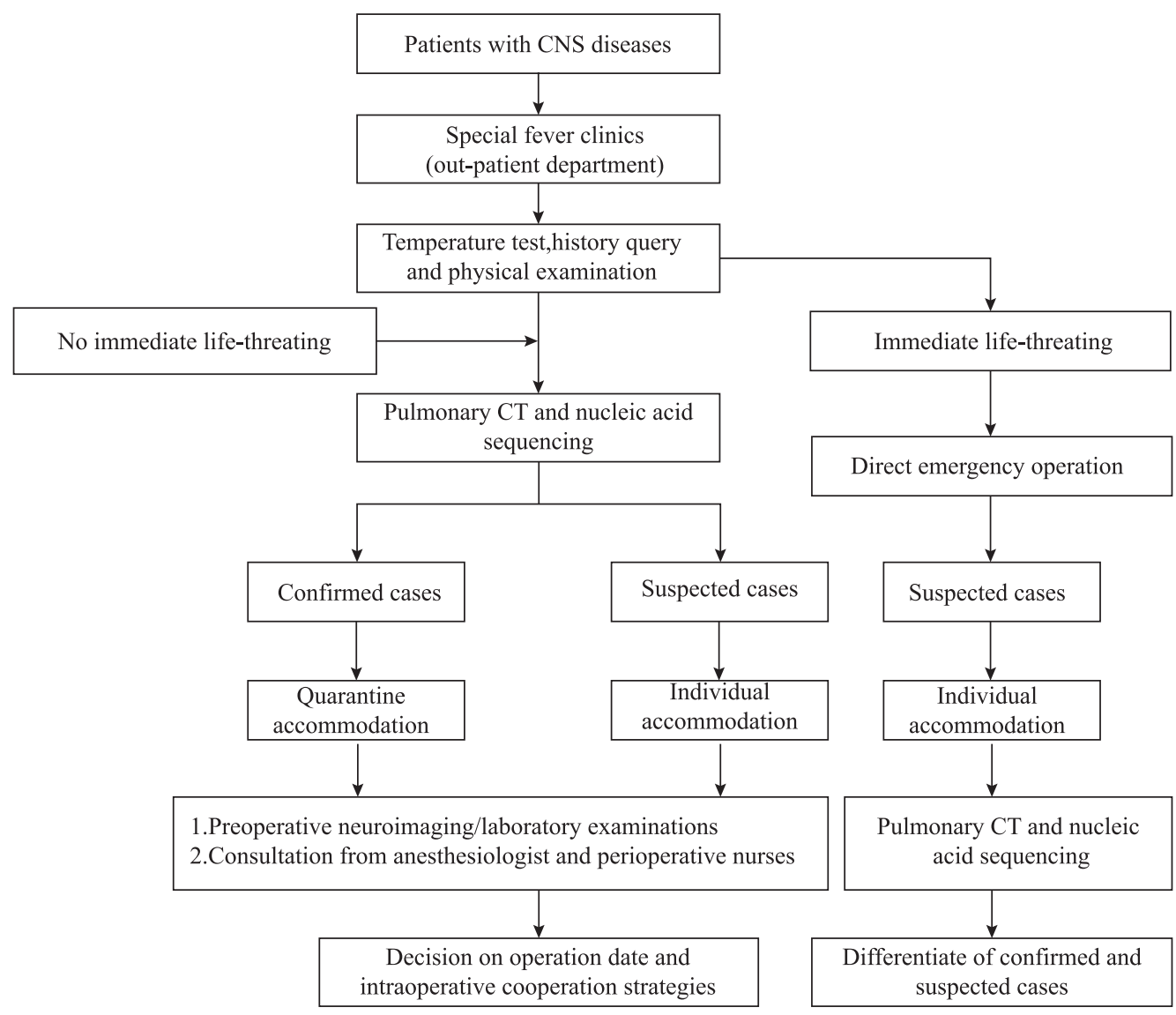

Fig. 1 Preoperative evaluation and management for patients with CNS diseases under COVID-19 situation 


\subsection{Intraoperative Management}

As mentioned above, the surgical strategies were decided preoperatively. Operations should be performed in special negative pressure suction room. The operation room was assumed to be polluted/infected area. On that day of surgery, neurosurgeons performed strict sterilization and wore third level protection, then entered the operative room with anesthesiologists and perioperative nurses for preparation of subsequent surgical procedures. Meanwhile, patients were applied to necessary protection by special medical assistant and then transferred to the waiting room of operative unit, where the patients were handed over to perioperative nurses.

After anesthetic intubation, surgical sterilization was performed by neurosurgeons. All surgical clothes, surgical kit and surgical shields should be disposable items. Senior neurosurgeons were preferred to shorten the operation duration. For complicated operations, one or two neurosurgeons should be backed up for rotation. Double surgical gloves were recommended for neurosurgeons to avoid infection due to glove ruptures. We suggested that the speed of drilling should be slowed to reduce skull bone aerosols. All surgical procedures should be accomplished according to preoperative strategies to reduce intraoperative bleeding and to shorten the operation duration.

After the operations were finished, the patients were transferred to the waiting room of operative unit, where the special medical assistant took over and subsequently transferred the patients back to the neurosurgery ward. All postoperative patients should be regarded as suspected cases and should be quarantined for at least 2 weeks (fig. 2).

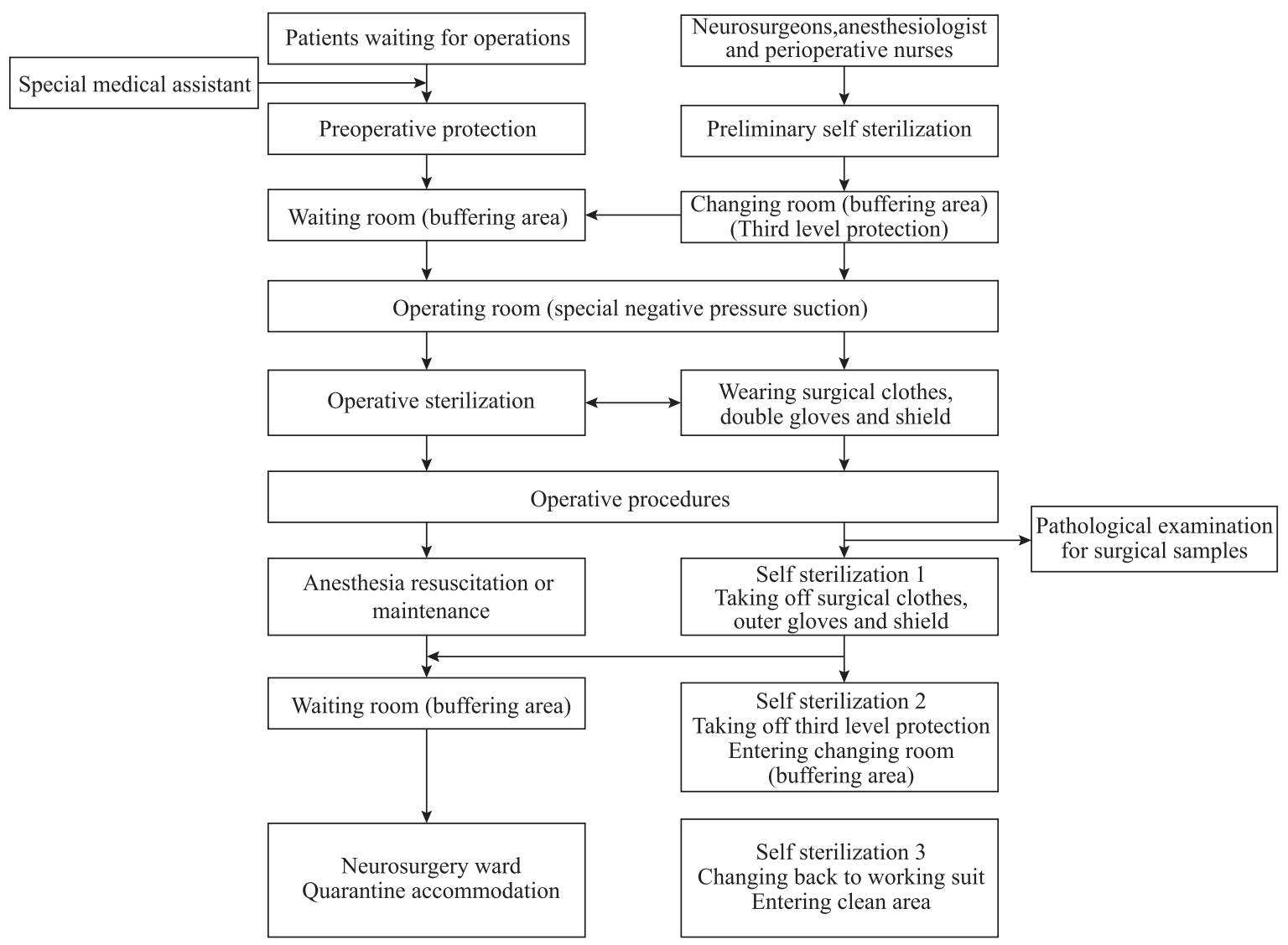

Fig. 2 Intraoperative management of patients with CNS disease under COVID-19 situation

\subsection{Postoperative Management}

All postoperative patients should be assumed to be suspected cases and quarantined for at least 2 weeks. Pulmonary CT scan and nucleic acid sequencing of throat swab should be repeated at least 3 times (in 2 weeks) after operation. The conditions of most postoperative patients of neurosurgery were critical. The monitoring and ventilator were necessary equipment for postoperative supportive care. The air ducts of ventilator should be daily replaced. Nutrition support was important for maintaining immunological function and reducing the possibility of virus and bacterial infection. If the pulmonary CT scan and nucleic acid sequencing of throat swab were negative for COVID-19 after 2 weeks, the quarantine could be terminated and patients were transferred to patient rooms of suspected cases. The recovery patients without COVID-19 would be transferred to neurosurgery recovery ward located on another floor. 


\section{CONCLUSIONS}

The outbreak of COVID-19 raises great challenges for medical work. Currently there is a lack of definite and effective treatment. The medical workers and public should be cautious in preventing and controlling the disease. The condition of patients with CNS emergency diseases is usually critical. As the largest neurosurgery department in central epidemic area, we integrate and redistribute the present resources to adapt the crisis situation. The recommendations above are drafted on the basis of our clinical experience and the latest relevant guidelines. We hope these proposals will be helpful to other neurosurgery staff. The suggestions and corrections from colleagues will be welcomed. With all the efforts, I do believe that we will finally win in this 'white war' against COVID-19.

\section{Conflict of Interest Statement}

None.

\section{REFERENCES}

1 Chen N, Zhou M, Dong X, et al. Epidemiological and clinical characteristics of 99 cases of 2019 novel coronavirus pneumonia in Wuhan, China: a descriptive study. Lancet, 2020,395(10223):507-513

2 Wu F, Zhao S, Yu B, et al. A new coronavirus associated with human respiratory disease in China. Nature, 2020. [Epub ahead of print].

3 Pan X, Ojcius DM, Gao T, et al. Lessons learned from the 2019-nCoV epidemic on prevention of future infectious diseases. Microbes Infect, 2020. [Epub ahead of print].

4 Sun $\mathrm{P}, \mathrm{Lu} \mathrm{X}, \mathrm{Xu} \mathrm{C}$, et al. Understanding of COVID-19 based on current evidence. J Med Virol, 2020. [Epub ahead of print].

5 Zhao WM, Song SH, Chen ML, et al. The 2019 novel coronavirus resource. Yi Chuan, 2020,42(2):212-221

6 Lu R, Zhao X, Li J, et al. Genomic characterisation and epidemiology of 2019 novel coronavirus: implications for virus origins and receptor binding. Lancet, 2020, 395(10224):565-574

7 Ralph R, Lew J, Zeng T, et al. 2019-nCoV(Wuhan virus), a novel Coronavirus: human-to-human transmission, travel-related cases, and vaccine readiness. J Infect Dev Ctries, 2020,14(1):3-17

8 Wang D, Hu B, Hu C, et al. Clinical Characteristics of 138 Hospitalized Patients With 2019 Novel CoronavirusInfected Pneumonia in Wuhan, China. JAMA, 2020. [Epub ahead of print].

9 Lai CC, Shih TP, Ko WC, et al. Severe acute respiratory syndrome coronavirus 2 (SARS-CoV-2) and coronavirus disease-2019 (COVID-19): The epidemic and the challenges. Int J Antimicrob Agents, 2020:105924

10 Ai T, Yang Z, Hou H, et al. Correlation of Chest CT and RT-PCR Testing in Coronavirus Disease 2019 (COVID-19) in China: A Report of 1014 Cases. Radiology, 2020:200642

11 Shanmugaraj B, Malla A, Phoolcharoen W.Emergence of Novel Coronavirus 2019-nCoV: Need for Rapid Vaccine and Biologics Development. Pathogens, 2020,9(2):148-150

12 Kwok KO, Wong V, Wei VWI, et al. Novel coronavirus (2019-nCoV) cases in Hong Kong and implications for further spread. J Infect, 2020. [Epub ahead of print]. (Received Feb. 29, 2020; revised Mar. 3, 2020) 